D.T. Wickramasinghe, L. Ferrario, and G.V. Bicknell, eds.

\title{
Modeling Tidal Effects in Accretion Discs
}

\author{
Patrick Godon \\ Jet Propulsion Laboratory, California Institute of Technology, MS \\ 238-332, 4800 Oak Grove Dr., Pasadena, CA 91109, USA
}

\begin{abstract}
A two-dimensional time-dependent spectral code is used for the study of tidal effects in accretion discs. A cool disc around a white dwarf (characteristic of CV systems) is modeled under the assumption of a polytropic equation of state and a standard alpha viscosity prescription. For a mass ratio $q<0.1$ (considered here) and under the assumption of a reflective inner boundary, tidal effects induce an eccentric $(m=1$ azimuthal) mode in the disc together with an elliptic ( $\mathrm{m}=2$ azimuthal) mode in the inner disc.
\end{abstract}

\section{Introduction}

It is presently believed that tidal interaction of discs with an orbiting exterior companion can be responsible for eccentricity growth in systems with a low mass companion. However, while the theoretical analysis (Lubow 1991a) clearly shows the existence of the eccentric instability, there has been some controversy between the numerical results. Simulations using SPH numerical codes do reproduce the eccentricity growth in discs (Whitehurst 1988, 1994, Hirose \& Osaki 1990, Lubow 1991b, Murray 1996), while usual finite difference (either Eulerian or Lagrangian) codes do not show any sign of an eccentric mode in the disc (Sawada, Matsuda \& Hachisu 1986a \& b, Sawada et al. 1987, Spruit et al. 1987, Lin \& Papaloizou 1993, Różyczka \& Spruit 1993, Savonije, Papaloizou \& Lin 1994). Heemskerk (1994, using a finite difference method) has shown that the tidal resonance eccentricity instability grows when only the $m=3$ component of the perturbing potential of the companion is taken into account. When the full perturbing potential is included, the outer edge of the disc does not reach the $3: 1$ tidal resonance region and the eccentric instability does not grow. When numerical simulation are carried out, the initial density profile in the viscous disc has to re-arrange itself, and if the mass ratio of the binary is small enough, the disc will reach the $3: 1$ resonance radius. In the $\mathrm{SPH}$ codes, the alpha viscosity parameter scales like the vertical thickness of the disc $\alpha=\alpha(H)$, and increases outward. The viscosity in the outer disc is much larger than in the standard model ( $\alpha=$ constant) and could therefore cause the disc to spread to large radii on a small time scale.

In this short work, we show the results of a spectral numerical code. We found that an eccentric $(m=1)$ and an elliptic $(m=2)$ azimuthal modes form in the disc when the inner boundary is a rigid reflective boundary. However, when a free inner boundary condition is assumed (like in the simulations of the 
previous authors), the disc is steady and exhibits only a double spiral pattern co-rotating with the binary.

\section{Numerical modeling}

We solve the full Navier-Stokes equations in the plane of the disc, assuming a polytropic equation of states and a standard alpha viscosity prescription. The spatial dependence of the equations is treated with a hybrid FourierChebyshev Spectral method of collocation, and an explicit fourth order RungeKutta method is used for the temporal scheme. The outer open boundary is treated with non-reflective boundary conditions. The code was also tested with an isothermal equation of state, as well as with a more realistic EOS, including heating and cooling in the energy equation. The modeling was carried out in the inertial frame of reference as well as in the rotating frame of the binary. Cool discs (as found in CV systems) and hot disc (as found in T Tauri systems) were modeled. However, in the present work we show only the modeling of a cool disc, with a low mass ratio (as characteristic of CV systems exhibiting the superhump phenomenon). The equations are solved in the rotating frame of reference, using the polytropic version of the code. More details on the numerical method and the models can be found in Godon 1996.

\section{Results}

In the present model we chose $\alpha=0.1, H / r=0.05$ at the outer boundary, the polytropic index $n=3$, the mass ratio $q=0.08$, the inner boundary is placed at $R_{i n}=1$, the outer boundary at $R_{\text {out }}=5$, and the binary separation is $a=12$. The Mach number $\left(r \Omega / c_{s}\right)$ is in the range 20-40. In a first run the inner boundary is a free boundary, through which matter flows freely. In this case a double spiral patter (co-rotating with the binary) forms after one orbital period, and the disc stays in this steady state for up to more than 150 orbits (time over which the simulation was followed). In the very inner part of the disc, the density decreases due to the inner boundary condition. In a second run the inner boundary is a rigid boundary, rotating at the local Keplerian velocity. This inner boundary condition corresponds to a fast rotating accreting white dwarf. It is enough for the WD to accrete about $0.1 M_{\odot}$ to rotate near break-up (Narayan \& Popham 1989). This is true at least for these CVs which show no observational evidence of a boundary layer (see also Livio, this volume). Here also a double spiral forms during the first binary orbit. The double spiral wave propagates inward to small radii and reaches the inner boundary. In an inviscid disc the spiral wave is expected to grows as it propagates inward (Spruit 1989, see also Dgani, Livio and Regev 1994). However for the present value of alpha $(\alpha=0.1)$ the wave amplitude decreases inward. During the first binary orbit a one-armed spiral pattern emanating from the outer disc, propagates inward. This $m=1$ eccentric mode is reflected at the inner boundary. With time, this $\mathrm{m}=1$ trapped mode is amplified and dominates the whole disc. At about five binary orbital times an $m=2$ elliptic mode appears in the inner disc. This $m=2$ mode is characterized by an elliptic density pattern (the streamlines are elliptic) which makes a prograde precession with a period of 3 times the local Keplerian 

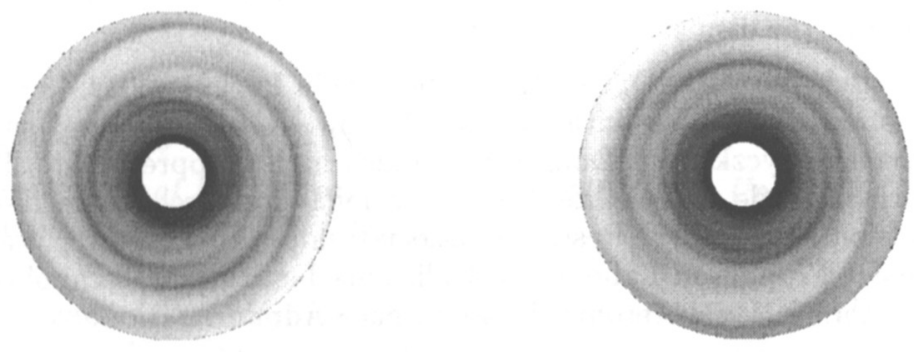

Figure 1. Grayscale of the density showing the $m=1$ eccentric pattern, a one armed-spiral pattern, superposed to the double spiral wave. Two snapshots are shown.
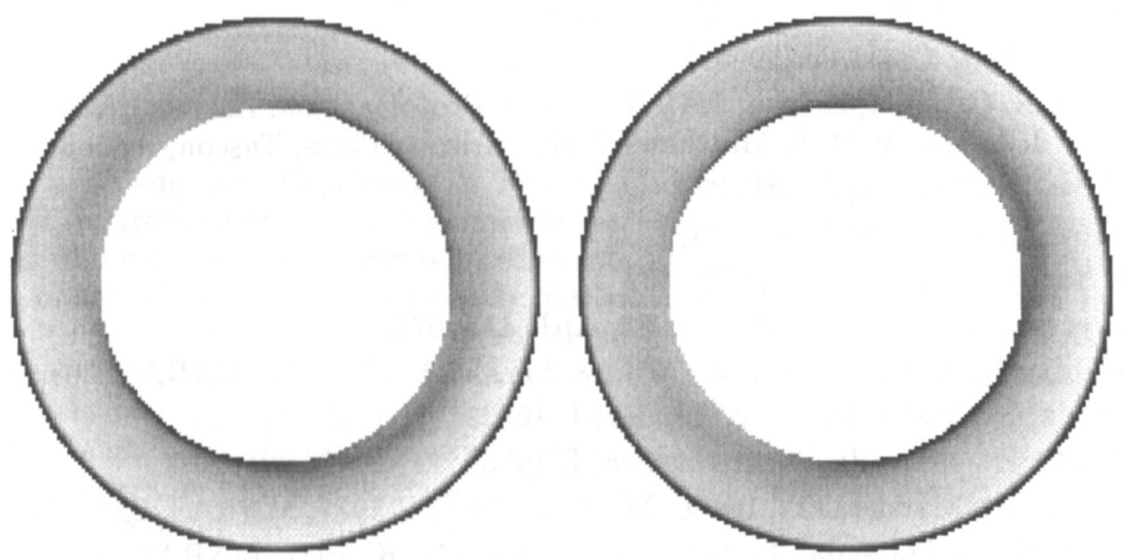

Figure 2. Grayscale of the density showing the $\mathrm{m}=2$ elliptic pattern in the inner disc. Two snapshots are shown.

period (or $1 / 13$ the binary period). The period of precession correspond well to the period of short period oscillations observed in the inner disc of CVs (Warner 1986). The eccentric $m=1$ mode induces local eccentricity in the disc. The local eccentric patterns make a retrograde precession with a period of the order of the binary period. In Figure 1 we show the grayscale of density patterns in the disc. The local eccentric patterns can clearly be seen. In Figure 2 the grayscale of the density is shown in the inner part of the disc. The contrast has been enhanced to show the elliptic density pattern near the inner boundary. By the end of the run (about 35 orbital periods) the disc is not globally eccentric. At this stage of the simulations, it is not clear whether the $m=1$ eccentric mode is related to the $3: 1$ tidal resonance eccentric instability or not. The $3: 1$ resonance radius is located slightly outside the computational domain at about $r \approx 5.5$, More simulations are being carried out and will be presented elsewhere (Godon 1996). We intend to check the effects of different important parameters: the location 
of inner and outer boundaries (and their ratio), the viscosity prescription, the sound speed in the disc and the mass ratio.

Acknowledgments. During the conference in Port Douglas, I enjoyed several interesting discussions with Stephen Lubow. Comments and suggestions from Michael Różyczka and Doug Lin are also highly appreciated. This work was performed while the author held a National Research Council - (NASA Jet Propulsion Laboratory) Research Associateship. This research was carried out at the Jet Propulsion Laboratory, California Institute of Technology, under contract to the National Aeronautics and Space Administration.

\section{References}

Dgani, R., Livio, M., \& Regev, O. 1994, ApJ, 436, 270

Godon, P. 1996, ApJ, submitted

Heemskerk, M. H. M. 1994, A\&A, 288, 807

Hirose, M., \& Osaki, Y. 1990, PASJ, 42, 135

Lin, D. N. C., \& Papaloizou, J. C. B. 1993, in Protostars and Planets III, E. Levi, J. Lunine \& M. S. Matthews, Univ. Arizona Press, Tuscon, Arizona, 749

Lubow, S. 1991a, ApJ, 381, 259

Lubow, S. 1991b, ApJ, 381, 268

Murray, J. 1996, MNRAS, 279, 402

Różyczka, M., \& Spruit, H. C. 1993, ApJ, 417, 677

Savonije, G. J., Papaloizou, J. C. B., \& Lin, D. N. C., 1994, MNRAS, 268, 13

Sawada, K., Matsuda, T., \& Hachisu, I. 1986a, MNRAS, 219, 75

Sawada, K., Matsuda, T., \& Hachisu, I. 1986b, MNRAS, 221, 679

Sawada, K., Matsuda, T., Inoue, M., \& Hachisu, I. 1987, MNRAS, 224, 307

Spruit, H. C., Matsuda, T., Inoue, M., \& Sawada, K. 1987, MNRAS, 229, 517

Spruit, H. C. 1989, in Theory of Accretion Disks, F. Meyer et al., Dodrecht, Kluwer, 325

Warner, B. 1986, Ap\&SS, 118, 271

Whitehurst, R. 1988, MNRAS, 232, 35

Whitehurst, R. 1994, in Theory of Accretion Disks-2, W. J. Duschl, J. Frank, F. Meyer, E. Meyer-Hofmeister \& W. M. Tscharnuter, Dodrecht, The Netherlands, 135

\section{Discussion}

S. Lubow: A double Fourier transform (in the azimuthal direction and in time) can be carried out on the density to verify to what extent is the $m=1$ mode observed in the simulations related to the tidal resonance eccentric instability. 